\title{
Journal of ovarian research reviewer acknowledgement 2014
}

Sham S Kakar ${ }^{1 *}$, Stefano Palomba², Benjamin K Tsang ${ }^{3}$ and David T Curiel ${ }^{4}$

\section{Contributing reviewers}

The editors of Journal of Ovarian Research would like to thank all of our reviewers who have contributed to the journal in Volume 7 (2014).

Abban-Mete Gülcin

Turkey

Alviggi Carlo

Italy

Amorim Christiani

Belgium

Angioli Roberto

Italy

Anschau Fernando

Brazil

Archer Gary

United States of America

Argenta Peter

United States of America

Arisan Elif Damla

Turkey

Aydin Yusuf

Turkey

Baka Stavroula

Greece

\section{Bao Shisan \\ Australia}

Barbolina Maria

United States of America

Barua Animesh

United States of America

Bashiri Asher

Israel

Bast Robert

United States of America

Bellis Susan

United States of America

\section{Bermudez Yira}

United States of America

Bevilacqua Estela

Brazil

Bhartiya Deepa

India

Bidzinski Mariusz

Poland

\author{
Bosenberg Marcus \\ Brazil \\ Branchini Gisele \\ Brazil \\ Bukovsky Antonin \\ Czech Republic \\ Büldt Georg \\ Germany \\ Buxton David \\ United States of America \\ Cannon Mark \\ United States of America \\ Cao Cong \\ China \\ Capodanno Francesco \\ Italy \\ Catteau-Jonard Sophie \\ France \\ Cecconi Sandra \\ Italy
}

\footnotetext{
* Correspondence: ovarianresearch@biomedcentral.com

'Department of Physiology and Biophysics, University of Louisville, Louisville, KY 40202, USA

${ }^{2}$ Department of Obstetrics, Gynecology and Pediatrics, Sterility Centre P. Bertocchi, Obstetrics and Gynecology Unit, A.O. S. Maria Nuova, IRCCS, Reggio Emilia and University of Modena, Reggio Emilia, Italy

${ }^{3}$ Department of Obstetrics \& Gynaecology, University of Ottawa and the

Chronic Disease Program, Ottawa Hospital Research Institute, Ottawa K1H

8 L6, Canada

${ }^{4}$ Cancer Biology Division, School of Medicine, Washington University in St. Louis, St.

Louis, MO 63108, USA
}

\section{() Biomed Central}


Cedar Marcelle I.

United States of America

Cheema Birinder

Australia

Chen Shuo

China

Chen Mei-jou

Taiwan

Chian Ri-Cheng

Canada

Christenson Lane

United States of America

Christman Gregory

United States of America

Chudecka-Glaz Anita

Poland

Cognasse Fabrice

France

Constantinou Pamela

United States of America

Corleta Helena

Brazil

Cosar Emine

Turkey

Dahan Michael

Canada

Dane Banu

Turkey

Davis John S.

United States of America

De Leo Vincenzo

Italy

DiFeo Analisa

United States of America

Donnez Jacques

Belgium

Dorsett Martin Wanda A

American Samoa

Downs Stephen

United States of America

Dursun Polat

Turkey

Eckstein Niels

Germany
Emoto Makoto

Japan

Er Tze Kiong

Taiwan

Ercan Cihangir Mutlu

Turkey

Evans Aco

Ireland

Fainzilber Mike

Iran

Falbo Angela

Italy

Feng Lingda

China

Ferrari Renata

Brazil

Fiorillo Lucia

Italy

Folgueira Maria Aparecida A Koike

Brazil

Foster Warren

Canada

Furlong Fiona

United Kingdom

Garcia Angel

Spain

Garcia Kara

United States of America

Geller Melissa

United States of America

Genazzani Alessandro

Italy

Georgopoulos Neoklis

Greece

Ghezzi Fabio

Italy

Goel Poonam

India

Gokce Cumali

Turkey

Gomez Raul

Spain

Grabowski Andrzej

Poland
Gu Jianren

China

Guedes Alexis

Brazil

Gungor Tayfun

Turkey

Guo Hua

United States of America

Gupta Digant

United States of America

Han Gencheng

China

Hashizume Kazuyoshi

Japan

Hawkridge Adam

United States of America

Hayashi Masahiro

United States of America

Hiraike Osamu

Japan

Hohenstein Peter

United Kingdom

Homburg Roy

Israel

Hovatta Outi

Sweden

Hsu Ming-I

Taiwan

Hua Jinlian

China

Huang HeFeng

China

Huhtinen Kaisa

Finland

Humaidan Peter

Denmark

Huntsman David

Canada

Iavazzo Christos

Greece

Irving-Rodgers Helen

Australia

Isidoro Ciro

Italy 
Jana Barbara

Poland

Jiang Zhongli

China

Jiao Ze-Xu

United States of America

Jindal Sangita

United States of America

Johnson Joshua

United States of America

Kakar Sham

United States of America

Kantara Carla

United States of America

Kar Sujata

India

Karpf Adam

United States of America

Karuppaiyah Selvendiran

United States of America

Katabuchi Hidetaka

Japan

Katiyar Santosh

United States of America

Kenigsberg Shlomit

Canada

Kim Won Ho

South Korea

Kim Yun Hwan

South Korea

Kim Tae Joong

South Korea

Kiss-Toth Endre

United Kingdom

Knapp Pawel

Poland

Kobayashi Hiroshi

Japan

Koumarianou Anna

Greece

Krause Diane

United States of America

Kreeger Pamela

United States of America
Krzysztof Szyllo

Poland

Kucukgoz Gulec Umran

Turkey

Kumaramanickavel Govindasamy

India

Kurihara Hiroaki

Japan

Kushnir Vitaly

United States of America

Kyritsis Athanassios

Greece

La Sala Giovanni Battista

Italy

Le Tien

Canada

Leader Arthur

Canada

Ledger William

Australia

Lee Keun Ho

South Korea

Levy Tally

Israel

Li Xuelian

China

Lim Carol

United States of America

Lipay Monica

Brazil

Liu Ping

China

Ma Jinfeng

China

Machado Santelli Glaucia M

Brazil

Maggio da Fonseca Angela

Brazil

Mahner Sven

Germany

Mandato Vincenzo Dario

Italy

Mandel (molinas) Nil

Turkey
Mariani Luciano

Italy

Markman Maurie

United States of America

Markowska Janina

Poland

Masters Seth

Australia

Mateizel Ileana

Belgium

Matos Maria Helena

Brazil

Mayer Barbara

Germany

Meng Ryan

China

Mes-Masson Anne-Marie

Canada

Messinis Ioannis

Greece

Minegishi Takashi

Japan

Mitchell Gillian

Australia

Miyano Takashi

Japan

Moncef Benkhalifa

France

Moreno Barb

Italy

Morgante Giuseppe

Italy

Murphy Bruce

Canada

Muscogiuri Giovanna

Italy

Nakamura Yasuhiko

Japan

Nam Joo-Hyun

South Korea

Nassir Mani

Germany

Ng Ernest

Hong Kong 


\author{
Nicoli Alessia \\ Italy
}

Nilsson Eric

United States of America

Ohmichi Masahide

Japan

Okada Kiyoshi
Japan

Oprea-Ilies Gabriela $\mathbf{M}$

United States of America

Orio Francesco

Italy

Orisaka Makoto
Japan
Orvieto Raoul
Israel
Osuga Yutaka
Japan
Öztürk Turhan Nilgün
Turkey
Pados George
Greece

Palanimuthu Ponnusamy Moorthy

United States of America

Palmer Julia

United Kingdom

Palomba Stefano

Italy

Patankar Manish

United States of America

Patel Divya

United States of America

Pathy Sushmita

India

Paz Filho Gilberto

Australia

Pearl Michael

United States of America

Pejovic Tanja

United States of America

Pelosi Emanuele

United States of America

Peng Chun

Canada
Penzes Peter

United States of America

Piltonen Terhi

Finland

Pocard Marc

France

Porpora Maria Grazia

Italy

Poveda Andres

Spain

Rasekh Jahromi Athar

Iran

Ratajczak Mariusz

United States of America

Reiter Russel

United States of America

Richard Francois

Canada

Richards JoAnne

United States of America

Rivkin Saul

United States of America

Robert Claude

Canada

Rodgers Ray

Australia

Rodriguez Ana Beatriz

Spain

Rose Peter

United States of America

Roy Debarshi

United States of America

Rueda Bo

United States of America

Ruggeri Rosaria

Italy

Sairam M Ram

Canada

Sakumoto Ryosuke

Japan

Salih Sana

United States of America

Salvetti Natalia

Argentina
Samant Rajeev

United States of America

Sanada Sakiko

Japan

Saraga-Babic Mirna

Croatia

Sawicki Wlodzimierz

Poland

Sayki Arslan Muyesser

Turkey

Scalici Jennifer

United States of America

Schattman Glenn

United States of America

Schouten Philip

Netherlands

Schultz Matthew

United States of America

Sellappan Selvaraju

India

Seow Kok-Min

Taiwan

Shimada Masayuki

Japan

Shoji Tadahiro

Japan

Shu Yimin

United States of America

Silambanan Santhi

India

Singh Ajay

United States of America

Singh Shailesh

United States of America

Smith Peter

New Zealand

Smith Steven C

Swaziland

Smitz Johan

Belgium

Songsasen Nucharin

United States of America

Sonoda Kenzo

Japan 
Sowter Heidi

United Kingdom

Speicher David

United States of America

Srivastava Rakesh

United States of America

Suganuma Nobuhiko

Japan

Sugino Norihiro

Japan

Sugiyama Toru

Japan

Sui Guangchao

United States of America

Sun Qing-Yuan

China

Sun Ying

United Kingdom

Tagliaferri Pierosandro Italy

Tanos Vasilios

Cyprus

Telleria Carlos

United States of America

Teruyoshi Saito

Japan

Tsai Eing-Mei

Taiwan
Tsang Benjamin

Canada

Tukiendorf Andrzej

Poland

Tüttelmann Frank

Germany

Uccella Stefano

Italy

Vaclavikova Radka

Czech Republic

Vanderhyden Barbara

Canada

Vincenzo De Leo

Italy

Virant-Klun Irma

Slovenia

Wang Junjie

China

Wang Qi

Canada

Wang Qi

United States of America

Wheeler Stephanie B

Austria

Wiest Jonathan

United States of America

Wojakowski Wojciech

Poland
Wong Alice

Hong Kong

Wong $\mathbf{P} \mathbf{C}$

Singapore

Xue Kai

China

Yavuzcan Ali

Turkey

Yazbek Joseph

United Kingdom

Yokoyama Yoshihito

Japan

Yoshida Junji

Japan

Yoshino Kiyoshi

Japan

Zeillinger Robert

Austria

Zeimet Alain

Austria

Zhang Xia

United States of America

Zullo Fulvio

Italy 